\title{
Combination therapy of interferon- $\alpha$ and 5-fluorouracil inhibits tumor angiogenesis in human hepatocellular carcinoma cells by regulating vascular endothelial growth factor and angiopoietins
}

\author{
HIROSHI WADA, HIROAKI NAGANO, HIROFUMI YAMAMOTO, ISAO ARAI, \\ HIDEO OTA, MASATO NAKAMURA, BAZARRAGCHAA DAMDINSUREN, TAKEHIRO NODA, \\ SHIGERU MARUBASHI, ATSUSHI MIYAMOTO, YUTAKA TAKEDA, KOJI UMESHITA, \\ YUICHIRO DOKI, KEIZO DONO, SHOJI NAKAMORI, MASATO SAKON and MORITO MONDEN \\ Department of Surgery, Graduate School of Medicine, Osaka University, \\ 2-2 Yamadaoka E-2, Suita 565-0871, Osaka, Japan
}

Received November 24, 2006; Accepted February 5, 2007

\begin{abstract}
We recently reported that interferon- $\alpha$ (IFN- $\alpha$ ) and 5-fluorouracil (5-FU) combination therapy in advanced hepatocellular carcinoma (HCC) achieved excellent clinical results. However, the mechanism underlying this combination therapy remains to be elucidated. In this study, we examined the anti-tumor effects of IFN- $\alpha$ and 5-FU combination therapy in vivo and aimed to reveal its anti-angiogenic effects by investigating the expression of vascular endothelial growth factor (VEGF) and angiopoietins (Ang-1 and Ang-2). Human HCC cells, HuH7, were subcutaneously injected in nude mice. Ten days later, groups of mice received treatment with IFN- $\alpha$ alone, 5-FU alone, or with a combination of IFN- $\alpha$ and 5-FU for four weeks. Immunohistochemical examinations of proliferating cell nuclear antigen (PCNA), cell differentiation antigen 34 (CD34), Ang-1, -2 and VEGF, terminal deoxynucleotidyl transferase-mediated dUTP nick end-labeling (TUNEL) assay and quantification of VEGF, Ang-1 and-2 mRNA using real-time RT-PCR were performed. Results
\end{abstract}

Correspondence to: Dr Hiroaki Nagano, Department of Surgery, Graduate School of Medicine, Osaka University, 2-2 Yamadaoka E-2, Suita 565-0871, Osaka, Japan

E-mail: hnagano@surg2.med.osaka-u.ac.jp

Abbreviations: 5-FU, 5-fluorouracil; Ang, angiopoietin; b-FGF, basic fibroblast growth factor; ELISA, enzyme-linked immunosorbent assay; FdUMP, fluorodeoxyuridine monophosphate; HCC, hepatocellular carcinoma; IFN, interferon; IL-8, interleukin-8; MMP, matrix metalloprotease; MVD, microvessel density; PCNA, proliferating cell nuclear antigen; PVTT, portal vein tumor thrombus; TUNEL, terminal deoxynucleotidyl transferase-mediated dUTP nick end-labeling; TRAIL, tumor necrosis factor-related apoptosis inducing ligand; VEGF, vascular endothelial growth factor

Key words: hepatocellular carcinoma, angiopoietin, angiogenesis, interferon, chemotherapy showed that IFN- $\alpha$ and 5-FU combination therapy significantly inhibited the growth of human HCC cells compared with the control group or single agent treatment. The combination therapy decreased PCNA-positive cells as well as microvessel density (MVD) and induced apoptosis of (TUNEL-positive cells) more than other treatment groups. Immunohistochemical analysis revealed that the combination therapy significantly decreased the expression of VEGF and Ang-2 and increased that of Ang-1. The ANG2/ANG1 mRNA expression ratio was significantly lower in the combination therapy group. In conclusion, our results suggested that IFN- $\alpha$ and 5-FU combination therapy has anti-proliferative and anti-angiogenic effects and can induce apoptosis in vivo. The synergistic and anti-angiogenic effects may in part be attributable to the regulation of Ang-1, -2 and VEGF.

\section{Introduction}

Hepatocellular carcinoma (HCC) is one of the most common malignancies worldwide (1) and the fourth highest cause of cancer-related death in Japan. The development of new diagnostic modalities has brought about an earlier diagnosis of small HCC and new therapeutic modalities, such as microwave coagulation therapy and radiofrequency ablation therapy, have improved the prognosis of patients with small HCC. However, the prognosis of patients with advanced HCC, for example those with portal vein tumor thrombus (PVTT) or intrahepatic metastasis, is quite poor and a standard treatment regimen for advanced HCC has not yet been established (2). Chemotherapy is commonly used for the treatment of various malignancies. However, it is not suitable for HCC because of its resistance to anti-cancer drugs (3).

The interferons (IFNs) are a family of natural glycoproteins and regulatory cytokines with pleiotropic cellular functions, such as anti-viral, anti-proliferative and immunomodulatory activities (4-6). Furthermore, previous reports indicate that IFN- $\alpha$ and IFN- $\beta$ have anti-angiogenic activities and downregulate the expression of pro-angiogenic molecules (7-12). The efficiency of IFN therapy for various malignancies has been investigated in several clinical trials and the results 
indicate that it can be effective against some angioproliferative diseases and vascularized malignancies (13-15). In HCC, the results of IFN- $\alpha$ monotherapy are not satisfactory and its effects remain controversial (16). However, in combination with other anti-cancer drugs, promising results were reported by several investigators (17-20). In a series of studies, we also reported recently the excellent clinical efficiency of IFN- $\alpha$ and 5-fluorouracil (5-FU) combination therapy for advanced HCC with PVTT and intrahepatic metastasis (21-24). The exact mechanism of action of this combination therapy is still unclear. IFN- $\alpha$ enhanced the expression of thymidine phosphorylase in colon cancer cells and the accumulation of fluorodeoxyuridine monophosphate (FdUMP) by inhibition of thymidylate in leukemia cells (25). We previously showed that the expression of the IFN- $\alpha / \beta$ receptor correlated with the growth-inhibitory activity of IFN- $\alpha$ and that IFN- $\alpha$ and 5-FU synergistically inhibited cell proliferation, induced cell cycle arrest $(26,27)$ and induced apoptosis by regulating the expression of apoptosis-related molecules (28). We also reported that IFN- $\alpha$ exerted immunomodulatory properties and that tumor necrosis factor-related apoptosis inducing ligand (TRAIL) and its receptor pathway, partially contributed to the anti-tumor effects of IFN- $\alpha$ and 5-FU combination therapy (29).

The present study was designed to further explore the mechanism of action of IFN- $\alpha / 5-\mathrm{FU}$ combination therapy in HCC. For this purpose, we established an in vivo nude mouse model of HCC and examined the effect of the treatment on the expression of vascular endothelial growth factor (VEGF) and angiopoietins (Ang-1 and Ang-2).

\section{Materials and methods}

Cell line and culture conditions. The hepatocellular carcinoma cell line HuH7 was maintained as an adherent monolayer in Dulbecco's modified Eagle's medium (DMEM) supplemented with $10 \%$ fetal bovine serum (FBS) and $1 \%$ penicillinstreptomycin mixture. Cell cultures were grown on plastic plates and incubated at $37^{\circ} \mathrm{C}$ in a mixture of $5 \% \mathrm{CO}_{2}$ and $95 \%$ air.

Reagents. Purified human IFN- $\alpha$ was obtained from Otsuka Pharmaceutical Co. (Tokushima, Japan) and purified 5-FU was obtained from Kyowa Hokko Co. (Tokyo, Japan). We used the following primary antibodies; polyclonal rabbit anti-human VEGF antibody (Santa Cruz Biotechnology, Santa Cruz, CA), polyclonal goat anti-human Ang-1 antibody (Santa Cruz, CA), polyclonal goat anti-human Ang-2 antibody (Santa Cruz, CA), monoclonal mouse anti-human proliferating cell nuclear antigen (PCNA) antibody cloned PC-10 (Dako, Glostrup, Denmark) and polyclonal rat anti-mouse cell differentiation antigen 34 (CD34) antibody (BD Biosciences, Flanklin Lakes, NJ).

Subcutaneous xenograft model in nude mice. Specific, pathogen-free, female athymic nude mice (BAL B/c nu/nu, 4- to 6-week-old) were purchased from CLEA Japan, Inc. (Tokyo, Japan). The mice were maintained under specific pathogen-free conditions in accordance with the institutional guidelines of animal care. HuH7 cells were uniformly seeded into $15 \mathrm{~cm}$ dishes and after reaching 80-90\% confluence, were briefly treated with $0.25 \%$ trypsin and $0.02 \%$ ethylenediaminetetraacetic acid (EDTA). Trypsinization was stopped with a medium containing 10\% FBS (fetal bovine serum). The cells were washed once with free medium and then resuspended in free medium. The cells $\left(5 \times 10^{6}\right.$ cells $/ 0.1 \mathrm{ml}$ DMEM) were subcutaneously injected in the right flank of each mouse. The IFN/5-FU combination therapy was started after growth of the tumor to $5-7 \mathrm{~mm}$ in diameter (10 days after the injection of cells). The dose of IFN- $\alpha$ was based on the results of previous studies $(12,30)$ and was adjusted so as to match the schedules of IFN- $\alpha$ used recently in clinical studies $(20,23)$. The doses and schedules of 5-FU represent the widely used standard clinical regimen (31-33). Mice were randomly assigned to one of the four groups as follows; (a) mice of the first group were administered a subcutaneous (SC) injection of IFN- $\alpha$ (20,000 units/body) three times per week, (b) mice of the second group were administered an intraperitoneal (IP) injection of 5-FU (30 mg/kg) three times per week, (c) mice of the third group were admministered an SC injection of IFN- $\alpha$ (20,000 units/body) and an IP injection of 5-FU $(30 \mathrm{mg} / \mathrm{kg}$ ) three times per week and (d) mice of the fourth group were administered SC and IP injections of phosphate buffered saline for the control group three times per week. There were eight mice in each group. Tumor volume was measured twice a week and was calculated using the following formula; (longest diameter) x (shortest diameter $)^{2}$ x 0.5 . Four weeks after the initial treatment, all mice from each group were sacrificed and tumors were harvested for examination. One part of the tumor was fixed in $10 \%$ buffered formalin for immunohistochemical staining, the other part was embedded in optimal cutting temperature (OCT) compound for frozen sectioning and stored at $-80^{\circ} \mathrm{C}$. The remainder of the tumor was later placed in RNA (Qiagen, Hilden, Germany) for RNA isolation.

Immunohistochemistry detection of PCNA, VEGF, Ang-1 and Ang-2. Formalin-fixed paraffin-embedded sections were used for immunohistochemical identification of PCNA, VEGF, Ang-1 and Ang-2. Sections measuring $5 \mu \mathrm{m}$ in thickness were deparaffinized in xylene and rehydrated in a graded series of ethanol baths. The immunostaining procedure was performed using Vectastain ABC peroxidase kits (Vector Labs, Burlingame, CA) as described previously (34). Briefly, after deparaffinization and rehydration, the sections were treated with an antigen retrieval procedure in $0.01 \mathrm{M}$ sodium citrate buffer ( $\mathrm{pH} \mathrm{6.0)} \mathrm{for} 40 \mathrm{~min}$ at $95^{\circ} \mathrm{C}$ and then incubated in methanol containing $0.3 \%$ hydrogen peroxide for $20 \mathrm{~min}$ at room temperature to block endogenous peroxidase. All primary antibodies; mouse anti-PCNA (diluted 1:400), rabbit anti-VEGF (diluted 1:100), goat anti-Ang-1 (diluted 1:50) and goat anti-Ang-2 (diluted 1:50), were incubated overnight at $4^{\circ} \mathrm{C}$. After the sections were incubated with biotinylated secondary antibody and peroxidase-conjugated streptavidin, peroxidase reactions were developed with 3,3'-diaminobenzidine tetrachloride (Wako Pure Chemical Industries). For a positive control, we used tissue of a placenta, which expressed VEGF, Ang-1 and Ang-2 proteins (35), was incubated in each staining procedure. For the negative control, nonimmunoreactive rabbit IgG or Tris-buffered saline were 




Figure 1. Effect of IFN- $\alpha / 5-F U$ therapy in nude mice (Volume of tumor, $\mathrm{V}=\mathrm{L} \mathrm{x} \mathrm{W}$ /2). HuH7 cells were subcutaneously injected into nude mice ( $\mathrm{n}=8$, each group). Treatment was initiated when tumors grew to a size of 5-7 mm in diameter (10 days after injection of cells). Nude mice were not treated ( $)$, treated by SC injection of IFN- $\alpha(20,000$ units/mouse) alone ( $\bullet$ ), treated by IP injection of 5-FU (30 mg/kg) alone $(\bigcirc)$, or treated by a combination of IFN- $\alpha$ and $5-\mathrm{FU}$ $(\diamond)$. Data are mean volume of tumors calculated by the following formula; (longest diameter) x (shortest diameter) 2 x 0.5 . Data are mean \pm SEM. The tumor volume of the combined therapy group was significantly decreased compared with other groups $(\mathrm{p}<0.05)$.

applied instead of the primary antibody. The intensity of immunohistochemical staining of VEGF, Ang-1 and Ang-2 was evaluated using MacSCOPE software (Mitani corp., Japan). For quantification of cell proliferation, five microscopic fields were randomly selected at high power magnification (x200) and the average counts of PCNA-positive cells were determined.

TUNEL assay. To detect apoptosis, we used the terminal deoxynucleotidyl transferase-mediated dUTP nick endlabeling (TUNEL) method, using the Apop Tag in situ apoptosis detection Kit (Chemicon International, Inc., Temecula, CA) as described previously (27). This method can detect fragmented DNA ends of apoptotic cells. Briefly, the paraffin-embedded sections were deparaffinized in xylene and rehydrated in a graded series of ethanol baths. The sections were treated with $20 \mu \mathrm{g} / \mathrm{ml}$ of proteinase $\mathrm{K}$ in distilled water for $10 \mathrm{~min}$ at room temperature and in methanol containing $0.3 \%$ hydrogen peroxide for 20 min to block endogenous peroxidase. The remaining procedures were performed according to the instructions provided by the manufacturer. For the quantification of apoptosis, five microscopic fields were randomly selected at high power magnification (x200) and the average counts of TUNEL-positive cells were calculated.

Identification of microvessel density by CD34 immunohistochemistry. Frozen sections ( $8 \mu \mathrm{m}$ thickness) were fixed in cold acetone for $10 \mathrm{~min}$ at $-20^{\circ} \mathrm{C}$. The sections were washed in PBS three times for $5 \mathrm{~min}$ each and were incubated in methanol with $0.3 \%$ hydrogen peroxide for endogenous peroxidase block. Subsequent procedures were the same as for paraffin-embedded sections. Rat polyclonal anti-mouse CD34 antibody (diluted 1:20, BD Bioscience, San Jose, CA) was used as the primary antibody for the detection of tumor vessels. Ten microscopic fields were randomly selected at x100 magnification and the average counts of CD34-positive vessels were determined as the microvessel density (MVD) of an individual tumor.

RNA extraction and quantitative real-time RT-PCR. Total RNA was extracted from frozen tissues via a single step method using TRIzol reagent (Life Technologies, Gaithersburg, MD). Total RNA (1 $\mu \mathrm{g})$ was used for reverse transcription and complementary DNA (cDNA) was generated using the Reverse transcription system (Promega, Madison, WI) as described previously (30). Quantification of mRNA expression of VEGF, ANG1 and ANG2 was performed using a real-time thermal cycler, Light Cycler $^{\circledR}$ and detection system (Roche Diagnostics, Mannheim, Germany). For detection of the amplification products, LightCycler-DNA master SYBR green I (Boehringer Mannheim, Mannheim, Germany) was used as described previously (29). Briefly, a $20 \mu 1$ reaction volume containing $2 \mu \mathrm{l}$ of cDNA and $0.2 \mu \mathrm{mol} / 1$ of each primer was applied to a glass capillary. The primers used were as follows; human VEGF (forward, 5'-AAGCCATCCTGTG TGCCCCTGATG-3'; reverse, 5'-GCGAATTCCTCCTGCC CGGCTCAC-3'), human ANG1 (forward, 5'-AAATGGAA GGAAAACACAAGGAA-3'; reverse 5'-ATCTGCACAGT CTCTAAATGGT-3'), human ANG2 (forward, 5'-GACGGC TGTGATGATAGAAATAGG-3'; reverse, 5'-GACTGTAG TTGGATGATGTGCTTC-3') and human $\beta$-actin (forward, 5'-GAAAATCTGGCACCACACCTT-3'; reverse, 5'-GTTG AAGGTAGTTTCGTGGAT-3'). PCR cycle conditions were set as described previously (35). The annealing temperatures of ANG1, ANG2, VEGF and $\mathrm{B}$-actin were $53^{\circ} \mathrm{C}, 51^{\circ} \mathrm{C}, 56^{\circ} \mathrm{C}$ 
A
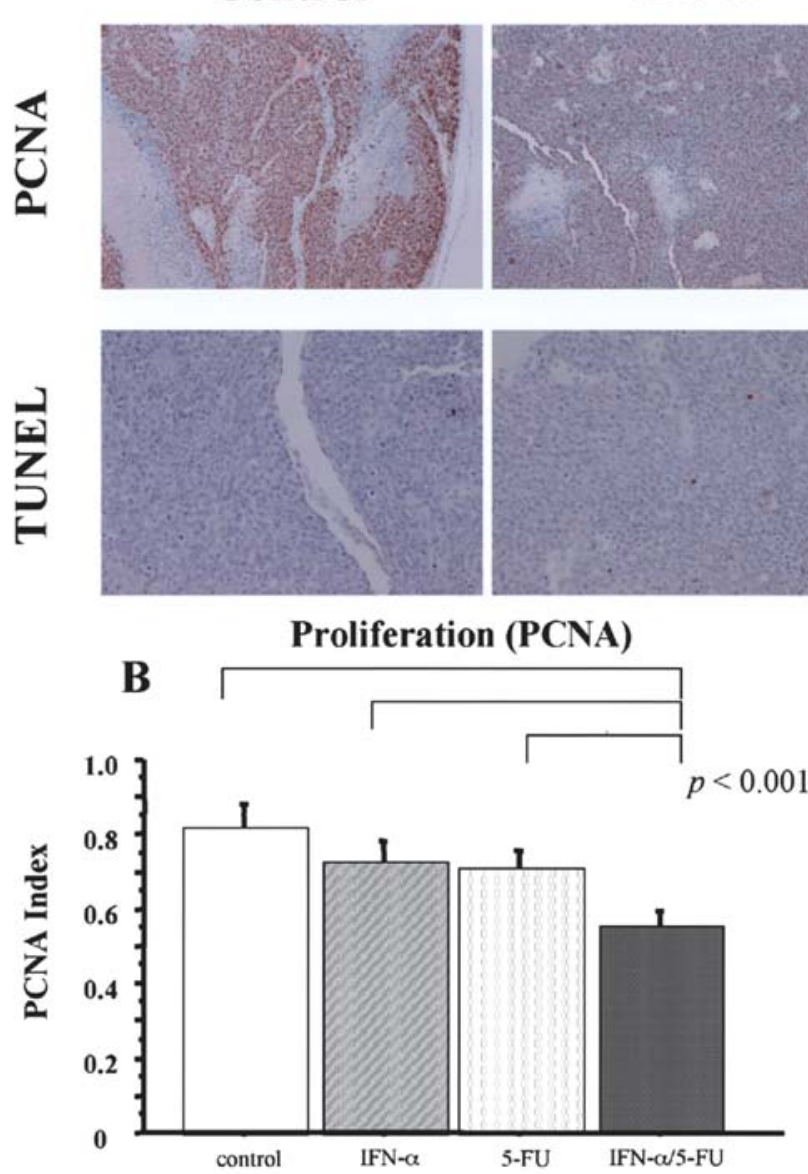

IFN- $\alpha$
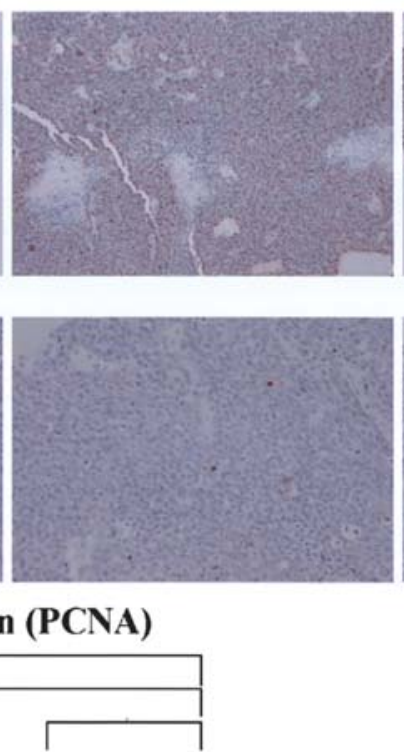
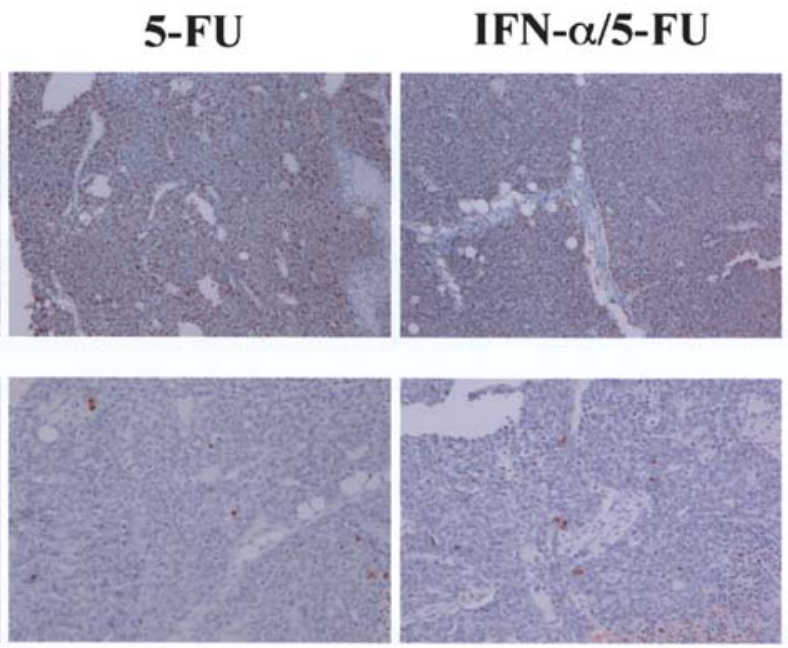

C

Apoptosis (TUNEL)

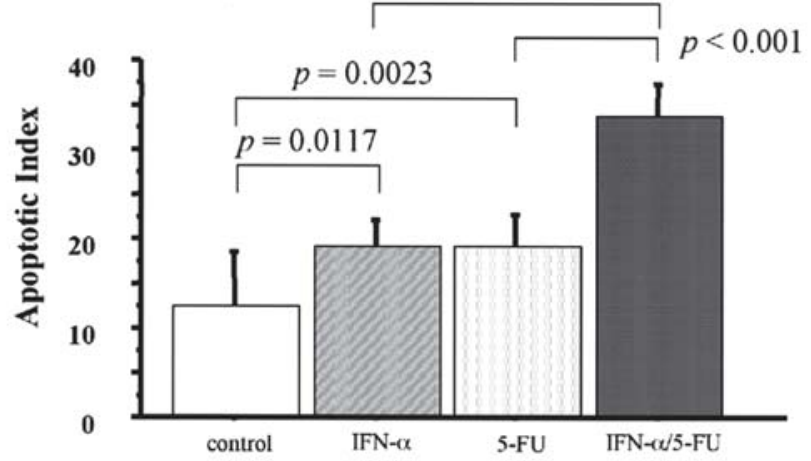

Figure 2. (A) Immunohistochemical analyses of PCNA (cell proliferation) and TUNEL (cancer cell apoptosis). Twenty-seven days after initial treatment, tumors were harvested from control mice or mice treated with IFN- $\alpha$ (20,000 units/mouse) alone, treated with 5 -FU (30 mg/kg) alone or treated with a combination of IFN- $\alpha$ and 5-FU. (B) Cell proliferation in each treatment group. Quantification of cell proliferation was expressed as the percentage of total cancer cells per field that were PCNA-positive in 5 random microscopic fields at high power magnification (x200). Numbers represent mean \pm SD. In combined therapy groups, the percentage of PCNA-positive cells was significantly decreased compared with the other groups ( $<<0.001)$. (C) Apoptosis of cancer cells. For quantification of apoptosis, the average number of TUNEL-positive cells was calculated in 5 random microscopic fields at high power magnification (x200). Data are mean \pm SD. In the group treated with IFN- $\alpha$ or 5-FU alone, the number of TUNEL-positive cells was increased versus control ( $\mathrm{p}=0.0117$ and 0.0023 , respectively). Apoptosis was significantly induced in tumors of mice of the combination therapy group, versus the other groups $(\mathrm{p}<0.001)$.

and $58^{\circ} \mathrm{C}$, respectively. A quantitative analysis of mRNA was performed using LightCycler ${ }^{\circledR}$ analysis software (Roche Diagnostics). The expression level of each angiogenic factor was normalized to the level of $\beta$-actin mRNA. We compared the ratio of ANG1/ß-actin, ANG2/ß-actin, VEGF/ß-actin and ANG2/ANG1 between each treatment group.

Statistical analysis. Data are expressed as mean \pm SD or SEM. Statistical analysis was performed using the StatView J-4.5 program (Abacus Concepts, Inc., Berkeley, CA). The tumor volume of each treatment group was compared by ANOVA. The unpaired Student's t-test was used to examine the difference in cell proliferation, apoptosis, MVD and expression of VEGF, Ang-1, Ang-2 proteins and an mRNA ratio between each group. A p-level $<0.05$ was considered statistically significant.

\section{Results}

IFN- $\alpha$ and 5-FU combination therapy for HCC xenografts. The growth curve of the implanted tumor in each group is shown in Fig. 1. On day 27, the tumor volume of the control group was $3.8 \pm 1.2 \mathrm{~cm}^{3}$ and those of the single agent IFN- $\alpha$ and 5-FU groups were $2.8 \pm 1.6$ and $2.5 \pm 1.2 \mathrm{~cm}^{3}$ (mean \pm SEM), respectively. While the single agent therapy reduced the tumor volume compared with the control group, these differences were not statistically significant. The tumor volumes of the combined therapy group were $1.4 \pm 0.4 \mathrm{~cm}^{3}$ and were significantly smaller in size than those of the other groups $(\mathrm{p}<0.05)$. The body weights of mice after removing xenografts on the 27 th day in the control, IFN- $\alpha$ alone, 5-FU alone and the combination group were $14.8 \pm 1.2,14.3 \pm 2.6$, $14.3 \pm 1.5$ and $14.4 \pm 1.8 \mathrm{~g}$, respectively (mean $\pm \mathrm{SD}$ ). There were no significant differences between the weight of the mice in each group.

IFN- $\alpha$ and 5-FU combination therapy inhibits tumor cell proliferation and angiogenesis and induces apoptosis. Examining cell proliferation, PCNA-positive cells in the control group was $81.6 \%$, while the percentage with IFN- $\alpha$ or 5-FU treatment alone was $72.5 \%$ and $70.8 \%$, respectively. In the combination therapy group, the cell proliferation was 
Table I. MVD and the expression of angiogenic factors in each treatment.

\begin{tabular}{llllc}
\hline Group & MVD & VEGF & Ang-1 & Ang-2 \\
\hline Control & $29.6 \pm 2.9$ & $35.4 \pm 3.2$ & $18.8 \pm 4.3$ & $25.5 \pm 1.3$ \\
IFN- $\alpha(20,000$ units $)$ & $18.1 \pm 2.9^{\mathrm{a}}$ & $22.3 \pm 8.2^{\mathrm{a}}$ & $23.6 \pm 7.1$ & $19.8 \pm 3.3^{\mathrm{a}}$ \\
$5-\mathrm{FU}(30 \mathrm{mg} / \mathrm{kg})$ & $22.0 \pm 3.8^{\mathrm{a}}$ & $30.4 \pm 6.2$ & $30.2 \pm 2.4^{\mathrm{a}}$ & $15.0 \pm 9.1$ \\
IFN- $\alpha / 5-\mathrm{FU}$ & $10.3 \pm 2.1^{\mathrm{b}}$ & $15.1 \pm 7.6^{\mathrm{c}}$ & $41.5 \pm 5.7^{\mathrm{d}}$ & $8.8 \pm 8.6^{\mathrm{e}}$ \\
\hline
\end{tabular}

The data showed mean $\pm \mathrm{SD}$. ${ }^{\mathrm{a}} \mathrm{p}<0.05$ compared with tumors of control mice. ${ }^{\mathrm{b}} \mathrm{p}<0.001$ compared with tumors of control mice, mice treated with IFN- $\alpha$ or 5-FU alone. ${ }^{c} \mathrm{p}<0.003$ compared with tumors of control mice and $\mathrm{p}<0.03$ compared with tumors of mice treated with 5 -FU alone. ${ }^{\mathrm{d}} \mathrm{p}<0.02$ compared with tumors of control mice, mice treated with IFN- $\alpha$ or 5 -FU alone. ${ }^{\mathrm{e}} \mathrm{p}<0.02$ compared with tumors of control mice, mice treated with IFN- $\alpha$ alone.

significantly inhibited in comparison with control or single therapy groups, with a percentage of PCNA-positive cells of $55.4 \%$. The average number of TUNEL-positive cells at high power magnification (x200) in each treatment group; the control, IFN- $\alpha$ alone, 5-FU alone and combination of IFN- $\alpha$ and 5-FU was 12.4, 19.1, 19.2 and 33.7, respectively, indicating that the combination therapy induced significant apoptosis of tumor cells ( $\mathrm{p}<0.001)$ (Fig. 2).

The MVDs of tumors in the control group were 29.6 \pm 2.9 , in the IFN- $\alpha$ alone group $18.1 \pm 2.9$, in the 5-FU alone group $22.0 \pm 3.8$ and in the combination therapy group 10.3 \pm 2.1 , respectively. MVD was not significantly reduced in the group treated by 5-FU alone but was in the group treated by IFN- $\alpha$ alone or by the combination of IFN- $\alpha$ and 5-FU. Furthermore, MVD in the combined therapy group was significantly reduced relative to the other groups (Table I and Fig. 3).

Immunohistochemical analysis of angiogenic factors. We evaluated the protein expression of tumors in each treatment group by immunohistochemistry. Representative samples of immunohistochemical staining of Ang-1, Ang-2 and VEGF are shown in Fig. 3. The expression of Ang-2 and VEGF were significantly decreased in tumors of mice treated with IFN- $\alpha$ and 5-FU compared with tumors of control mice or from mice treated with IFN- $\alpha$ or 5-FU alone. The expression of Ang-1 was significantly increased in tumors of the IFN- $\alpha$ and 5-FU combination therapy group (Table I).

ANG2/ANG1 mRNA expression ratio in tumors of mice treated with IFN- $\alpha$ and 5-FU. ANG1, ANG2 and VEGF mRNA levels in the combination therapy group were $2.17 \pm 1.66,1.13 \pm 0.78$ and $1.46 \pm 0.66$, respectively. ANG2 and VEGF mRNA levels in the combination therapy group were lower than that of IFN- $\alpha$ or 5-FU alone, but these differences were not significant. The ANG2/ANG1 mRNA expression ratio was significantly lower in the combination therapy group compared with the group treated by IFN- $\alpha$ alone or the control group (Fig. 4).

\section{Discussion}

In the present study, we investigated the mechanism of the anti-tumor effect of IFN- $\alpha$ and 5-FU combination therapy using a nude mouse xenograft model. The administration of IFN- $\alpha$ combined with 5 -FU three times per week significantly inhibited the growth of human hepatocellular carcinoma cells injected subcutaneously into nude mice. Interferon monotherapy or combination therapy with various chemotherapeutic agents in other solid malignancies is well documented in various in vivo models $(7,9,12,30)$. As reported previously, daily or three times weekly injections of IFN- $\alpha$ was necessary to produce therapeutic effects. With regard to the dosage, a total dose per week of 35,000 to 70,000 units of IFN- $\alpha$ inhibited tumor growth and angiogenesis of xenografts in nude mice (30). In HCC, Hisaka et al (36) reported that a subcutaneous injection of 10,000-1,000,000 units of IFN- $\alpha$ decreased tumor volume in vivo in a dose-dependent fashion. In the group with a daily administration of 10,000 units of IFN- $\alpha$, the volume of the xenograft of human HCC cells was reduced to about $60 \%$ of the control. Therefore, we determined that the schedule for treatment with IFN- $\alpha$ would be three times per week, since this was recently used clinically and the dose would be 20,000 units/body. The maximum tolerated dose of 5-FU in nude mice was $60 \mathrm{mg} / \mathrm{kg}$, in a schedule of three injections every 4 days (32). The standard and widely used regimen for $5-\mathrm{FU}$ is $20-50 \mathrm{mg} / \mathrm{kg}$ per injection and a total dosage per week of about $100 \mathrm{mg} / \mathrm{kg}(31,33)$. We determined that 5-FU would be administered IP three times per week at a dose of $30 \mathrm{mg} / \mathrm{kg}$. In our study, single agent treatment (SC injection of IFN- $\alpha$ or IP injection of 5-FU alone) inhibited tumor growth compared with the control group, but the difference was not significant. We confirmed that IFN- $\alpha$ and 5-FU combination therapy significantly inhibited tumor growth compared with other groups. However, an orthotopic model by placing the cells in the hepatic parenchyma might be necessary to reveal the mechanisms of anti-angiogenic effects of IFN/5-FU combination therapy. The dosage and schedule of IFN- $\alpha$ and 5-FU used in our study were standard, clinically used and the estimated volume of the tumors after using a combined therapy for 4 weeks was $38 \%$ of those of the control group. In another study of IFN- $\alpha$ monotherapy, comparatively higher doses of IFN- $\alpha$ were needed to reduce tumor volumes to half of those of the control group (36). These phenomena emphasize the high anti-tumor effects of IFN- $\alpha$ and 5-FU combination therapy.

Our results demonstrated a significant decrease in PCNA-positive proliferating cells and an increase in TUNEL- 
Control
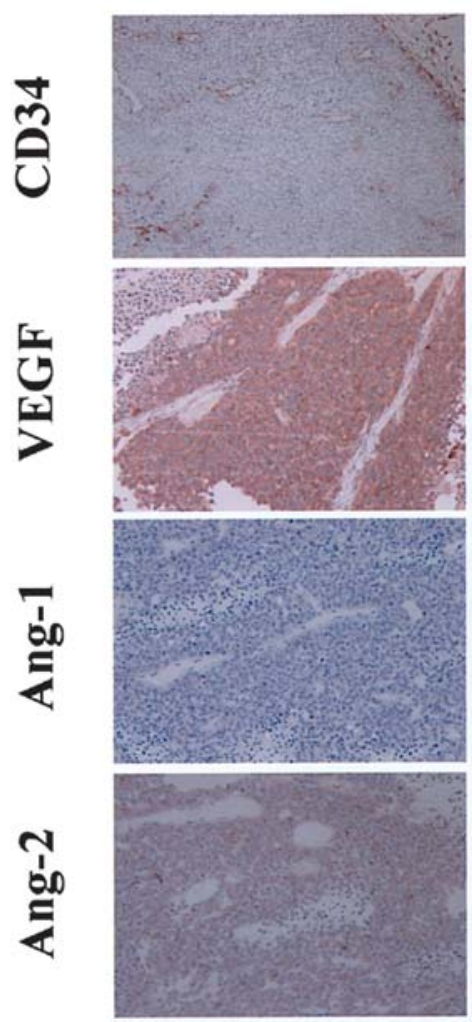

IFN- $\alpha$
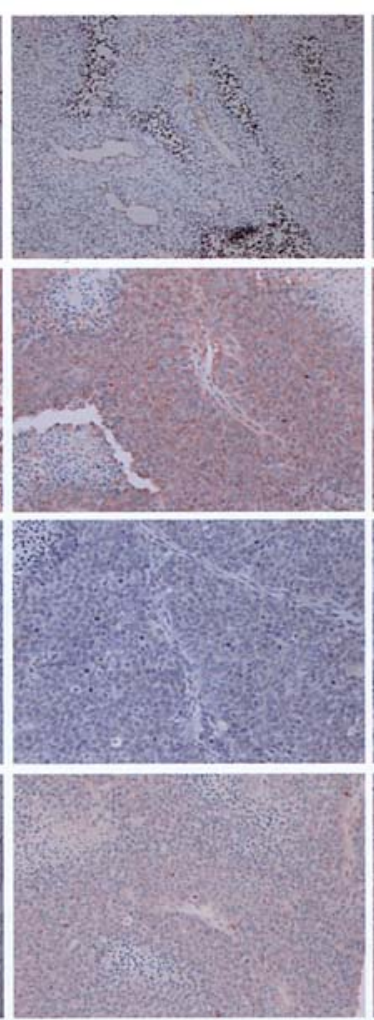

5-FU


IFN- $\alpha / 5-F U$
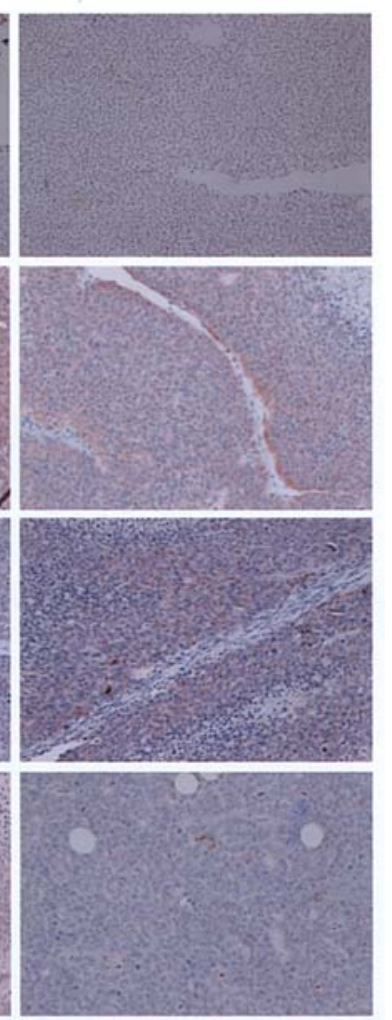

Figure 3. Immunohistochemical analyses of CD34 (endothelial cells) and VEGF, Ang-1 and Ang-2 (angiogenic factors). The sections were immunostained for expression of CD34 (to show MVD), VEGF, Ang-1 and Ang-2. Tumors of mice treated with combined IFN- $\alpha$ and 5-FU showed a significant decrease of MVD. The expression of VEGF and Ang-2 significantly decreased and the expression of Ang-1 significantly increased in tumors of combination therapy mice. Representative samples are shown (x100).

positive apoptotic cells in the combination therapy group, in agreement with our previous studies $(27,28)$. IFN- $\alpha$ has an anti-proliferative effect and the combination of IFN- $\alpha$ and 5-FU synergistically induces cell cycle arrest and up-regulation of p27Kip1 in vitro (27). In our recent study, the IFN- $\alpha$ and 5-FU combination therapy induced apoptosis and upregulated the expression of various apoptosis-regulated proteins, including Bcl-2, Bcl-xl and Bax (28). Kojiro et al reported that anti-proliferative effects of IFN- $\alpha$ and 5-FU in combination on a hepatocellular carcinoma cell line were attributable to the enhanced induction of S-phase arrest and apoptosis (37). These results are consistant with our present results.

We also examined the anti-angiogenic effects of IFN- $\alpha$ and 5-FU combination therapy, because angiogenesis is essential for tumor growth and metastasis (38) and HCC is one of the most hypervascular tumors. IFN- $\alpha$ has anti-angiogenic properties in clinical tumors such as Kaposi's sarcomas (15), infantile hemangiomas (13) and some vascular-rich malignancies, melanoma, renal cell carcinoma and neuroendocrine tumors (14). Immunohistochemical analysis showed a significant decrease in CD34-positive cells (and therefore MVD) in the combination treatment group. Both in vitro and in vivo, IFN- $\alpha$ inhibited the transcription and production of pro-angiogenic molecules. Previous studies showed that IFN- $\alpha$ decreased the production of major proangiogenic factors such as VEGF $(7,12)$, b-FGF (11), MMP-2 and MMP-9 (8,9), and IL-8 (10). Marschall et al (7) previously reported that the therapeutic effects of IFN- $\alpha$ on neuroendocrine tumor cells were based on Sp1- and/or Sp3-mediated inhibition of VEGF transcription both in vivo and in vitro. In pancreatic cancer cells, IFN- $\alpha$ combined with the chemotherapeutic agent gemcitabine, induced apoptosis of tumorassociated endothelial cells and decreased the local production of pro-angiogenic molecules from tumor cells (12).

The present data confirmed that the use of a combination therapy in an in vivo mouse model resulted in significant reductions in VEGF and Ang-2 protein expression and an increase in Ang-1 protein expression. We reported previously that cooperation between Ang-2 and VEGF plays an important role in enhancing the formation of new blood vessels in hepatic metastases of colorectal cancer (35). Furthermore, VEGF and Ang-2 have been shown to play an important role in angiogenesis in HCC, in our reports and those of others (34,39-41). Angiopoietins have been identified as a new family of endothelial growth factors and comprises ligands for the vascular endothelium-specific tyrosine kinase receptor Tie2 (42-44). Ang-1, which is an agonist of Tie2 and induces its phosphorylation, serves as a survival factor for endothelial cells and promotes recruitment of pericytes and smooth muscle cells. Therefore, Ang-1 is thought to help maintain and stabilize vascular networks (45). Ang-2 is a biological antagonist of Ang-1 and reduces vascular stability, blocking the stabilizing action of Ang-1. However, in the presence of 
A


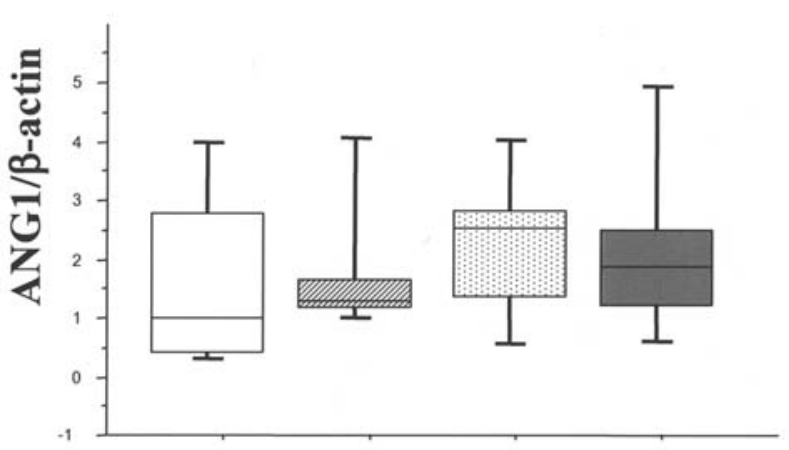

B

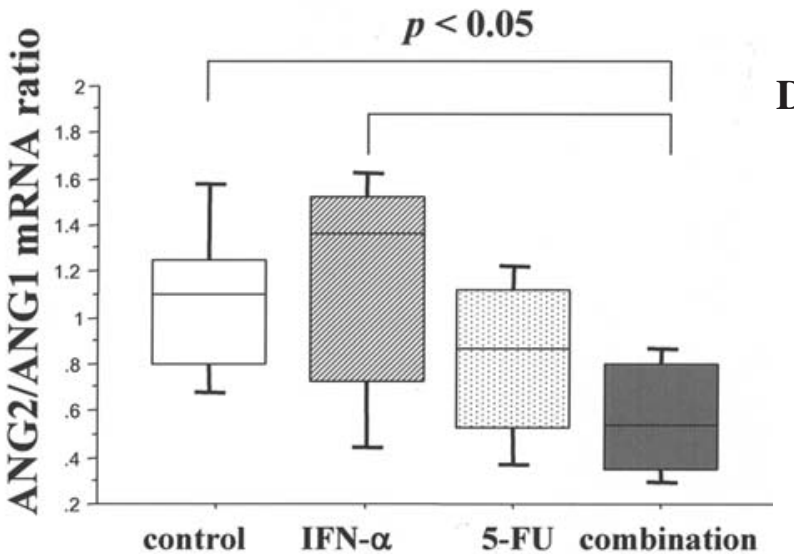

Figure 4. Expression of mRNA encoding VEGF, Ang-1 and Ang-2 and expression ratio of ANG2/ANG1 mRNA in tumors of control mice, mice treated with IFN- $\alpha$ alone, 5-FU alone and a combination of IFN- $\alpha$ and 5-FU. The mRNA expression levels were normalized to $\beta$-actin. Data are displayed in box plots, with mean values represented by the horizontal lines inside the boxes. Mean values are as follows: (A) VEGF: control, $1.51 \pm 0.90 ;$ IFN- $\alpha$ alone, $1.43 \pm 0.55 ; 5-\mathrm{FU}$ alone, 1.53 \pm 0.35 ; IFN- $\alpha / 5-F U, 1.46 \pm 0.66$; (B) ANG1: control, 1.64 \pm 1.53 ; IFN- $\alpha$ alone, 1.79 $\pm 1.35 ; 5-F U$ alone, $2.31 \pm 1.28 ;$ IFN- $\alpha / 5-F U, 2.17 \pm 1.66 ;(C)$ ANG2: control, $2.03 \pm 2.15 ;$ IFN- $\alpha$ alone, $1.89 \pm 1.02 ; 5-F U$ alone, $1.71 \pm 1.03$; IFN- $\alpha / 5-F U, 1.13 \pm 0.78 ; \mathrm{D}$, mRNA ratio of ANG2/ANG1: control, $1.08 \pm 0.37$; IFN- $\alpha$ alone, $1.15 \pm 0.24 ; 5-\mathrm{FU}$ alone, $0.83 \pm 0.34$; IFN- $\alpha / 5-\mathrm{FU}, 0.57 \pm 0.24$. Data are mean $\pm \mathrm{SD}$. The combination therapy resulted in a significant reduction of ANG2/ANG1 mRNA ratio compared with the control and IFN- $\alpha$ alone ( $\mathrm{p}=0.0087$ or 0.046 , respectively).

VEGF, Ang-2 induces vascular sprouting and angiogenesis (46). Ang-2 is markedly expressed in organs that undergo vascular remodeling, such as the ovaries and placenta (35). Furthermore, several studies reported similar findings in various malignancies including HCC and that the expression levels of Ang-2 protein and mRNA correlate with clinicopathological factors in HCC (39-41).

Our results showed that the combination therapy increased the mRNA levels of Ang-1 and decreased those of Ang-2. The difference in Ang-1 and Ang-2 levels in vivo was not significant. However, the Ang-2/Ang-1 mRNA ratio was significantly decreased by systemic administration of IFN- $\alpha$ and 5-FU. Although there is a discrepancy between the proteins and mRNA, the balance between Ang-1 and Ang-2 mRNA expressions is most important because the high Ang-2/ Ang-1 mRNA ratios in HCC were closely associated with portal vein invasion, tumor diameter, the MVD levels of HCC and the poor prognosis(41). The exact mechanism of regulation of angiopoietins remains unknown. IFN- $\alpha$ exerts most of its biological activity by altering the level of gene expression in target cells. IFN regulates oncogene expression resulting in the regulation of both transcriptional and posttranscriptional events (47). The transcriptional regulation of angiopoietins is not well characterized. Using ovarian cancer cells, Zhang et al (48) reported that tumor-derived VEGF upregulates Ang-2 in host stroma endothelial cells. Potente et al (49) recently reported that Ang-2 was exclusively regulated by forkhead box O (Foxo) 1. The Foxo subclass of transcriptional factors plays an important role in the control of cell growth, development and survival. Dephosphorylation of Foxo factors leads to the activation or repression of apoptosis- and cell cycle-related genes such as Bim, p27Kip1, MnSOD, or GADD45 $(50,51)$. We previously reported that the synergistic effect of IFN- $\alpha$ and 5-FU was in part attributable to alterations in cell cycle progression via up-regulation of p27Kip1 (27). We speculate that the IFN- $\alpha$ and 5-FU combined therapy may induce the regulation of angiopoietins, via regulation of Foxo, as well as up-regulation of p27Kip1. Further studies are needed to identify the mechanism of the transcriptional or post-transcriptional regulation of angiopoietins by IFN- $\alpha$ and 5-FU combined therapy.

In conclusion, we confirmed that the IFN- $\alpha$ and 5-FU combined therapy had anti-proliferative and anti-angiogenic effects and induced apoptosis, in human HCC cells using a nude mouse xenograft model. The synergistic and antiangiogenic effects of IFN- $\alpha$ and 5-FU may contribute to the anti-tumor effect against $\mathrm{HCC}$, through the regulation of VEGF and angiopoietins. 


\section{Acknowledgements}

This study was supported by a grant-in-aid for cancer research from the Ministry of Culture and Science of Japan.

\section{References}

1. Farmer DG, Rosove MH, Shaked A and Busuttil RW: Current treatment modalities for hepatocellular carcinoma. Ann Surg 219: 236-247, 1994.

2. Bruix $\mathbf{J}$ and Llovet JM: Prognostic prediction and treatment strategy in hepatocellular carcinoma. Hepatology 35: 519-524, 2002.

3. Shen DW, Lu YG, Chin KV, Pastan I and Gottesman MM: Human hepatocellular carcinoma cell lines exhibit multidrug resistance unrelated to MRD1 gene expression. J Cell Sci 98: 317-322, 1991.

4. Baron S and Dianzani F: The interferons: a biological system with therapeutic potential in viral infections. Antiviral Res 24: 97-110, 1994.

5. Gutterman JU: Cytokine therapeutics: lessons from interferon alpha. Proc Natl Acad Sci USA 91: 1198-1205, 1994.

6. Hertzog PJ, Hwang SY and Kola I: Role of interferons in the regulation of cell proliferation, differentiation, and development. Mol Reprod Dev 39: 226-232, 1994.

7. Von Marschall Z, Scholz A, Cramer T, et al: Effects of interferon alpha on vascular endothelial growth factor gene transcription and tumor angiogenesis. J Natl Cancer Inst 95: 437-448, 2003.

8. Gohji K, Fidler IJ, Tsan R, et al: Human recombinant interferonsbeta and -gamma decrease gelatinase production and invasion by human KG-2 renal-carcinoma cells. Int J Cancer 58: 380-384, 1994.

9. Huang SF, Kim SJ, Lee AT, et al: Inhibition of growth and metastasis of orthotopic human prostate cancer in athymic mice by combination therapy with pegylated interferon-alpha-2b and docetaxel. Cancer Res 62: 5720-5726, 2002.

10. Oliveira IC, Sciavolino PJ, Lee TH and Vilcek J: Downregulation of interleukin 8 gene expression in human fibroblasts: unique mechanism of transcriptional inhibition by interferon. Proc Natl Acad Sci USA 89: 9049-9053, 1992.

11. Singh RK, Gutman M, Bucana CD, Sanchez R, Llansa N and Fidler IJ: Interferons alpha and beta down-regulate the expression of basic fibroblast growth factor in human carcinomas. Proc Natl Acad Sci USA 92: 4562-4566, 1995.

12. Solorzano CC, Hwang R, Baker $\mathrm{CH}$, et al: Administration of optimal biological dose and schedule of interferon alpha combined with gemcitabine induces apoptosis in tumorassociated endothelial cells and reduces growth of human pancreatic carcinoma implanted orthotopically in nude mice. Clin Cancer Res 9: 1858-1867, 2003.

13. Castello MA, Ragni G, Antimi A, et al: Successful management with interferon alpha-2a after prednisone therapy failure in an infant with a giant cavernous hemangioma. Med Pediatr Oncol 28: 213-215, 1997.

14. Jonasch E and Haluska FG: Interferon in oncological practice: review of interferon biology, clinical applications and toxicities. Oncologist 6: 34-55, 2001.

15. Shepherd FA, Beaulieu R, Gelmon K, et al: Prospective randomized trial of two dose levels of interferon alfa with zidovudine for the treatment of Kaposi's sarcoma associated with human immunodeficiency virus infection: a Canadian HIV Clinical Trials Network study. J Clin Oncol 16: 1736-1742, 1998.

16. Llovet JM, Sala M, Castells L, et al: Randomized controlled trial of interferon treatment for advanced hepatocellular carcinoma. Hepatology 31: 54-58, 2000.

17. Leung TW, Patt YZ, Lau WY, et al: Complete pathological remission is possible with systemic combination chemotherapy for inoperable hepatocellular carcinoma. Clin Cancer Res 5: 1676-1681, 1999.

18. Urabe T, Kaneko S, Matsushita E, Unoura M and Kobayashi K: Clinical pilot study of intrahepatic arterial chemotherapy with methotrexate, 5-fluorouracil, cisplatin and subcutaneous interferon-alpha-2b for patients with locally advanced hepatocellular carcinoma. Oncology 55: 39-47, 1998.

19. Patt YZ, Hassan MM, Lozano RD, et al: Phase II trial of systemic continuous fluorouracil and subcutaneous recombinant interferon Alfa-2b for treatment of hepatocellular carcinoma. J Clin Oncol 21: 421-427, 2003.
20. Obi S, Yoshida H, Toune R, et al: Combination therapy of intraarterial 5-fluorouracil and systemic interferon-alpha for advanced hepatocellular carcinoma with portal venous invasion. Cancer 106: 1990-1997, 2006.

21. Miyamoto A, Umeshita K, Sakon M, et al: Advanced hepatocellular carcinoma with distant metastases, successfully treated by a combination therapy of alpha-interferon and oral tegafur/uracil. J Gastroenterol Hepatol 15: 1447-1451, 2000.

22. Sakon M, Nagano H, Dono K, et al: Combined intraarterial 5fluorouracil and subcutaneous interferon-alpha therapy for advanced hepatocellular carcinoma with tumor thrombi in the major portal branches. Cancer 94: 435-442, 2002.

23. Ota H, Nagano H, Sakon M, et al: Treatment of hepatocellular carcinoma with major portal vein thrombosis by combined therapy with subcutaneous interferon-alpha and intra-arterial 5fluorouracil; role of type 1 interferon receptor expression. $\mathrm{Br} \mathrm{J}$ Cancer 93: 557-564, 2005.

24. Nagano H, Sakon M, Eguchi H, et al: Hepatic resection followed by IFN-alpha and 5-FU for advanced hepatocellular carcinoma with tumor thrombus in the major portal branch. Hepatogastroenterology (In press).

25. Schwartz EL, Hoffman M, O'Connor CJ and Wadler S: Stimulation of 5-fluorouracil metabolic activation by interferonalpha in human colon carcinoma cells. Biochem Biophys Res Commun 182: 1232-1239, 1992.

26. Damdinsuren B, Nagano H, Sakon M, et al: Interferon-beta is more potent than interferon-alpha in inhibition of human hepatocellular carcinoma cell growth when used alone and in combination with anticancer drugs. Ann Surg Oncol 10: 1184-1190, 2003.

27. Eguchi H, Nagano H, Yamamoto $\mathrm{H}$, et al: Augmentation of antitumor activity of 5-fluorouracil by interferon alpha is associated with up-regulation of p27Kip 1 in human hepatocellular carcinoma cells. Clin Cancer Res 6: 2881-2890, 2000.

28. Kondo M, Nagano H, Wada H, et al: Combination of IFN-alpha and 5-fluorouracil induces apoptosis through IFN-alpha/beta receptor in human hepatocellular carcinoma cells. Clin Cancer Res 11: 1277-1286, 2005.

29. Yamamoto T, Nagano H, Sakon M, et al: Partial contribution of tumor necrosis factor-related apoptosis-inducing ligand (TRAIL)/TRAIL receptor pathway to antitumor effects of interferon-alpha/5-fluorouracil against hepatocellular carcinoma. Clin Cancer Res 10: 7884-7895, 2004.

30. Slaton JW, Perrotte P, Inoue K, Dinney CP and Fidler IJ: Interferon-alpha-mediated down-regulation of angiogenesisrelated genes and therapy of bladder cancer are dependent on optimization of biological dose and schedule. Clin Cancer Res 5: 2726-2734, 1999.

31. Teicher BA, Chen V, Shih C, et al: Treatment regimens including the multitargeted antifolate LY231514 in human tumor xenografts. Clin Cancer Res 6: 1016-1023, 2000.

32. Ishikawa Y, Kubota T, Otani Y, et al: Dihydropyrimidine dehydrogenase activity and messenger RNA level may be related to the antitumor effect of 5-fluorouracil on human tumor xenografts in nude mice. Clin Cancer Res 5: 883-889, 1999.

33. Britten CD, Hilsenbeck SG, Eckhardt SG, et al: Enhanced antitumor activity of 6-hydroxymethylacylfulvene in combination with irinotecan and 5-fluorouracil in the HT29 human colon tumor xenograft model. Cancer Res 59: 1049-1053, 1999.

34. Wada H, Nagano H, Yamamoto H, et al: Expression pattern of angiogenic factors and prognosis after hepatic resection in hepatocellular carcinoma: importance of angiopoietin-2 and hypoxia-induced factor-1 alpha. Liver Int 26: 414-423, 2006.

35. Ogawa M, Yamamoto $H$, Nagano $H$, et al: Hepatic expression of ANG2 RNA in metastatic colorectal cancer. Hepatology 39: 528-539, 2004.

36. Hisaka T, Yano H, Ogasawara S, et al: Interferon-alphaCon 1 suppresses proliferation of liver cancer cell lines in vitro and in vivo. J Hepatol 41: 782-789, 2004.

37. Kojiro S, Yano H, Ogasawara S, et al: Antiproliferative effects of 5 -fluorouracil and interferon-alpha in combination on a hepatocellular carcinoma cell line in vitro and in vivo. J Gastroenterol Hepatol 21: 129-137, 2006.

38. Folkman J: Tumor angiogenesis: therapeutic implications. N Engl J Med 285: 1182-1186, 1971.

39. Tanaka S, Mori M, Sakamoto Y, Makuuchi M, Sugimachi K and Wands JR: Biologic significance of angiopoietin-2 expression in human hepatocellular carcinoma. J Clin Invest 103: 341-345, 1999. 
40. Torimura T, Ueno T, Kin M, et al: Overexpression of angiopoietin- 1 and angiopoietin-2 in hepatocellular carcinoma. J Hepatol 40: 799-807, 2004.

41. Mitsuhashi N, Shimizu H, Ohtsuka M, et al: Angiopoietins and Tie-2 expression in angiogenesis and proliferation of human hepatocellular carcinoma. Hepatology 37: 1105-1113, 2003.

42. Maisonpierre PC, Suri C, Jones PF, et al: Angiopoietin-2, a natural antagonist for Tie2 that disrupts in vivo angiogenesis. Science 277: 55-60, 1997.

43. Davis S, Aldrich TH, Jones PF, et al: Isolation of angiopoietin-1, a ligand for the TIE2 receptor, by secretion-trap expression cloning. Cell 87: 1161-1169, 1996.

44. Suri C, Jones PF, Patan S, et al: Requisite role of angiopoietin-1, a ligand for the TIE2 receptor, during embryonic angiogenesis. Cell 87: 1171-1180, 1996.

45. Papapetropoulos A, Garcia-Cardena G, Dengler TJ, Maisonpierre PC, Yancopoulos GD and Sessa WC: Direct actions of angiopoietin-1 on human endothelium: evidence for network stabilization, cell survival and interaction with other angiogenic growth factors. Lab Invest 79: 213-223, 1999.
46. Asahara T, Chen D, Takahashi T, et al: Tie2 receptor ligands, angiopoietin-1 and angiopoietin-2, modulate VEGF-induced postnatal neovascularization. Circ Res 83: 233-240, 1998.

47. Harada H, Kitagawa M, Tanaka N, et al: Anti-oncogenic and oncogenic potentials of interferon regulatory factors- 1 and -2 . Science 259: 971-974, 1993.

48. Zhang L, Yang N, Park JW, et al: Tumor-derived vascular endothelial growth factor up-regulates angiopoietin-2 in host endothelium and destabilizes host vasculature, supporting angiogenesis in ovarian cancer. Cancer Res 63: 3403-3412, 2003.

49. Potente M, Urbich C, Sasaki K, et al: Involvement of Foxo transcription factors in angiogenesis and postnatal neovascularization. J Clin Invest 115: 2382-2392, 2005.

50. Tran H, Brunet A, Grenier JM, et al: DNA repair pathway stimulated by the forkhead transcription factor FOXO3a through the Gadd45 protein. Science 296: 530-534, 2002.

51. Kops GJ, Dansen TB, Polderman PE, et al: Forkhead transcription factor FOXO3a protects quiescent cells from oxidative stress. Nature 419: 316-321, 2002. 